a healthy person. With regard to the second case the dicease began when the sufferer was prostrated from a purely mechanical malady. When relief was given by an operation his health improved and he has remained well, although he has never become strong and continues to be unable to work on account of his physical infirmity and the pain produced by it. If the first of these cases stood alone there could hardly be any doubt about the disease being a constitutional one. In the second, however, any fresh systemic disturbance could not be well marked owing to its being implanted upon a man already debilitated by prolonged ill-health. His regaining health up to a certain point could be no reason why the disease with him should not be constitutional. Moreover, the fact that two persons in the same family were affected by the same complaint would point to a systemic rather than a local origin.

The treatment in these cases is eminently unsatisfactory, as little or nothing can be done except to build up the general health and to alleviate pain.

Ladbroke-grove, $W$.

\section{A CASE OF TOTAL EXTIRPATION OF THE PROSTATE.}

By G. P. NEWBOLT, F.R.C.S. ENG.,

HONORARY SURGEON AND DEAN OF CLINICAL SCHOOL, ROYAL SOUTHERN HOSPITAL, LIVERPOOL.

A MAN, aged 69: years, was admitted to the Royal Southern Hospital on April 15th last, suffering from retention of urine. His urinary trouble first began five years previously when an attack of retention necessitated the use of an instrument $\varepsilon$ nd c uring the last four years he had led a catheter life. He had to get up at night in order to relieve himself and had had several bad attacks of retention. On April 13th he was unable to pass his soft catheter, and on the 15 th he was admitted to hospital with a greatly distended bladder and a false passage from which he was bleeding freely. The bladder was aspirated above the pubes and four hours later a silver instrument was passed. After this the bladder was washed out twice daily, but the passage of the catheter caused him much pain and usually drew blood. On the 24th I saw him and passed a full sized silver prostatic catheter into his bladder; he had a large, soft, moveable prostate easily made out per rectum and bilaterally enlarged. The urine was acid, 1020, and contained blood and pus. On May 10th the patient's condition and the state of his urine having improved I determined to perform suprapubic prostatectomy. He was placed under chloroform and his bladder having been distended with ten ounces of boric lotion I opened the organ above the pubes and on introducing my lett index finger at once felt a large prostatic tumour which resembled the. uterus in shape with the cervix pointing upwards and backwards. Having scraped through the mucous membrane on the left side of the growth with my index finger-nail I was able to enucleate speedily the gland on that side below and from behind the triangular ligament. After spending some five minutes in trying to clear it from the urethra I tore the latter across on the vesical side of the triangular ligament and proceeded to free the right portion. This was more difficult and it was necessary to use scissors in order to free the under and upper portions of tbe right lobe from the base of the bladder. The growth was then seized with forceps and delivered through the suprapubic wound. Whilst operating the prostate was pushed up from the rectum by the fingers of a colleague. There was some hæmorrhage which was controlled by boric lotion at a temperature of $110^{\circ} \mathrm{F}$. A large rabber tube was put in the suprapubic wound and the rest of the incision was brought together with silkworm gut. There was some shock after the operation, from which the patient speedily rallied. The whole proceeding took 23 minutes from the first incision until the wound was sutured.

The after-history of the case is as follows: On the nex morning the patient's pulse and temperature were normal. The tube was removed from above the pubes in 48 hours and the bladder was irrigated daily through a soit catheter in the urethra. On the 20th, ten days after operation, 1 passed a full-sized silver catheter into the bladder and he passed a little urine the right way when his bowels acted on the night before. On June 8th he went home, the wound had closed and he passed urine freely three or four times in the day and once at night. He had an attack of eczema due to the irritation of the urine about the wound, but this speedily cleared up. Two weeks after his return home he had an attack of bronchitis when the wound opened and remained so for a fortnight when it healed soundly. He is now in good health, passes urine freely, and is quite comfortable. The prostate, which was entire, weighed five and three quarter ounces. The ejaculatory ducts were torn across and show well in the specimen. A very thin partition was felt between the rectum and the bladder after removal, but the gap left rapidly contracted. Microscopically the growth was fibro-adenoma. The operation so ably advocated by Freyer seems to be a most valuable one in suitable cases.

Liverpool.

\section{A CASE OF VERONAL POISONING.}

BY G. FERNANDEZ M. CLARKE, L.R.C.P. LOND., M. R.C.S. ENG., L.S.A. LOND.

THE following case may be of interest to the readers of THE LANCET.

At midday on Dec. 13th, 1903, I was telephoned for to see a woman who, from the message I received, was evidently suffering from narcotic poisoning. On arriving at the house I found my patient in what appeared to be a sound sleep. The breathing was deep and slightly stertorous; the pulse was slow but regular; the pupils reacted to light; and the temperature was normal. With some difficulty she could be aroused but relapsed immediately into her former state of semi-coma. The history which I obtained from her friends was that the patient's age was 19 years; she had been married only a few months and seemed to be very happy; she had had a "touch of the sun" when in Jersey, after which she had been subject to several hysterical attacks varying in intensity, and to the best of their knowledge she had not taken any drugs. With this information I decided not to resort to any drastic measures but to content myself with treating the symptoms. As time went on the symptoms gradually subsided and by midnight I was able to learn from her that she had taken something to make her sleep but of what cha racter that something was she absolutely refused to tell me. 1t was not until the next morning that I became acquainted with the drug in question when I received from her husband a box of veronal cachets, each containing e ght grains, which had been found in her room. I saw her again the same day, when she had apparently quite recovered, having slept sr undly throughout the previous night. On the 16th an erythematous rash appeared all over the body. There was a great amount of irritation of the skin, especially of the face which presented a very swollen appearance. There was little or no elevation of temperature and the symptoms passed off in the course of three days with the aid of sedative lotions. On the 21st I was again called to see the patient whom I found suffering from the following symptoms : tenderness in the right mastoid region with enlargement of the glands arourd; a thick ceruminous discharge from the right ear ; a temperature of $101^{\circ} \mathrm{F}$; the tongue strawberry coloured and pointed; and a pulse of 125 . She appeared to be in a very drowsy condition but assured me that she had not taken any more veronal. Refusing absolutely to take any form of liquid medicine I ordered her sulphate of quinine tabloids, each containing two grains to be taken every three hours. This I did more as a "placebo" than anything else. The next day, the $22 \mathrm{nd}$, the patient had periodical attacks of delirium alternating with periods of semi coma in which she lay on her back with her eyes half open. There was also a peculiar scarlatiniform rash over the face ard arms; the bowels were obstinately confined. On the 23rd the patient was about the same. Fearing the symptoms might be due to some obscure brain lesion (due to the sun attack in Jersey) I called in further advice but could not obtain any decided opinion. On the 24th the patient was still about the same, the temperature ranging from normal to $101^{\circ}$. Constipation was obstinate even after two enemata and half an ounce of castor oil in capsule had been given. Having great suspicion that the patient was still taking veronal by means of trickery I again impressed the husband to redouble his efforts to find out whether this 